\title{
The Primary Reactor Coolant System Concept of the Integral, Inherently-Safe Light Water Reactor
}

Matthew J Memmott ${ }^{1}$, Annalisa Manera² Jonathan Boyack ${ }^{1}$, Seth Pachecoํ․ Mingjun Wang ${ }^{2,4}$, Bojan Petrovic ${ }^{3}$,

1. Brigham Young University, 350 Clyde Building, Provo UT, 84602, 801-422-6237, memmott@byu.edu

2. University of Michigan, 2945 Cooley Building, Ann Arbor, MI 48109

3. Georgia Institute of Technology, 770 State Street, Atlanta, GA 30332

4. Xi'an Jiaotong University, No. 28 West Xianning Road, Xi'an 710049, China

Abstract - Nuclear power has enormous potential to provide clean, affordable baseload electricity worldwide. The events at Fukushima demonstrated, however, that nuclear safety must be enhanced in order to fully realize the latent potential of nuclear electricity. Small modular reactors, in particular, create significant safety benefits by eliminating large bore piping and the potential for a large-break loss of cooling accident (LOCA). The $I^{2} S$-LWR is a large-scale power plant concept designed to incorporate the integral reactor benefits previously exclusive to small modular reactors into large reactor systems. This paper presents and discusses the base design of the integral, inherently safe light water reactor $\left(I^{2} S\right.$ LWR) primary coolant system, highlighting the impact of five major design constraints and their impact on the design development. Key deviances from the primary coolant system for both current LWRs and $S M R s$ are indicated where appropriate, and key component design drawings of the I'S-LWR integral reactor pressure vessel (RPV) and supporting systems are provided. These include the reactor pressure vessel, reactor coolant pumps, the pressurizer, the microchannel heat exchangers, the decay heat removal exchangers, and the reactor vessel internals. The final integrated design of the primary coolant system described in this paper serves as the base design configuration for the $I^{2} S-L W R$, while component performance and analyses are described in companion papers.

\section{Keywords: Reactor Pressure Vessel; Integral Reactors, I2S-LWR, SMRs}

I. Introduction

Nuclear power has enormous potential to provide clean, affordable baseload electricity worldwide. The events at Fukushima demonstrated, however, that nuclear safety must be enhanced in order to fully realize the potential of nuclear electricity ${ }^{1}$. Recent developments in nuclear technology have resulted in "passive" safety systems capable of removing decay heat from a nuclear reactor without outside electricity or operator action for up to 3.5 days for large reactors ${ }^{2,3}$ and up to 7 days for small modular reactors $(\mathrm{SMR})^{4}$. Small modular reactors, in particular, create significant safety benefits by eliminating large bore piping and the potential for a large-break loss of cooling accident (LOCA). They also include a compact containment which minimizes core uncovery during events which actuate the automatic depressurization system (ADS). These SMRs, however, are limited to electricity production of under 300 MW. Therefore, it is of great interest to translate the enhanced safety of the SMRs to a larger sized reactor. The integral, inherently safe light water reactor $\left(I^{2} S-L W R\right)$ has been developed to satisfy this interest.

The integral, inherently safe light water reactor $\left(I^{2} S-L W R\right)$ is a large-scale power plant concept designed to incorporate the integral reactor benefits previously exclusive to small modular reactors into large reactor systems. The primary difference between prior integral reactor concepts (the SMRs) and the 
${ }^{2} \mathrm{~S}$-LWR is that the $\mathrm{I}^{2} \mathrm{~S}$-LWR design allows for the scale-up of electricity production capabilities to 1000 MWe while utilizing the integral design, thus benefiting from both economies of scale and integral reactor pressure vessel (RPV) enhancements. Additionally, the passive safety features of the I'S-LWR were designed to facilitate indefinite cooling, rather than the current 7 day limitation of the SMRs. In developing the $I^{2} S$-LWR, however, there are three primary challenges associated with scaling that need to be addressed.

First, the $I^{2} S$-LWR must remain cost competitive with current Gen III+ reactor systems 5 . The obvious impact of a power scale-up is that the reactor will have larger components and systems to accommodate the larger thermal power rating. This means that forgings sizes and concrete pour volumes will be increased. Ideally, a scaled-up design should attempt to minimize the raw materials used. Although economy of scale will provide some benefit for the I'S-LWR, there is the potential for cost benefits to be offset due to larger cost increases of key components where the cost scaleup is nonlinear. Additionally, equipment requirements should be minimized to reduce capital costs of the $I^{2} S$ LWR. Thus, the I'S-LWR design focuses on compact, combined, and simplified systems in order to minimize vessel and system volumes. Additionally, the vessel and components must be fabricated using current technologies.

Secondly, integral reactors often result in difficult maintenance due to the minimal free volume and awkward arrangement of equipment within both the containment and the RPV. In order to build utility company confidence in the mechanical design of the containment and RPV of the I'S-LWR, systems and components must be designed and incorporated in ways that minimize maintenance frequency and difficulty. In the $\mathrm{I}^{2} \mathrm{~S}$-LWR, components were designed so that maintenance requirements did not include additional specialized tools or systems relative to current light water reactors (LWRs), and so that maintenance can be accomplished within a 17 day refueling outage.

Third, increasing the output of an integral reactor to $1000 \mathrm{MWe}$ increases the volumetric heat output of the reactor vessel, thus making safe operation more difficult. This increase in volumetric heat generation is a result of limitations on RPV size due to fabrication constraints. As a result, the heat generation increases more than the RPV volume, which increases the volumetric heat generation of the primary system. This in turn challenges the capacity of the reactor to transfer heat from the core to the secondary system or external environment during normal and off-normal operation, respectively. Although compact heat exchangers solve this challenge during normal operation, accident performance can be negatively impacted. An additional primary focus of the ${ }^{2} \mathrm{~S}$-LWR design based on the higher volumetric heat output is to facilitate enhanced passive safety through the design of the systems, structures, and components. The $I^{2} S-L W R$ design team has developed several innovative technologies which were integrated into the RPV design to realize this goal without drastically altering the LWR licensing and commercialization pathways.

This paper presents and discusses the base design of the I'2S-LWR primary coolant system, highlighting the impact of five major design constraints and their impact on the design development. Key deviances from the primary coolant system for both current LWRs and SMRs are indicated where appropriate, and key component design drawings of the I'S-LWR integral RPV are provided.

\section{Primary Coolant System Design Criteria}


The development of the primary reactor coolant system is based upon several design criteria. Although these criteria are common to current light water reactors, their impact on the design can be quite different for integral reactors, primarily due to the compact RPV and containment sizes. The application of these design constraints directly impact the component development for the primary coolant system design, and thus these design criteria are referenced when component and system selections are made. The design criteria selected to guide the design of the integral reactor pressure vessel of the $I^{2} S-L W R$ are:

1. All components must fit within the reactor pressure vessel with sufficient peripheral spacing to perform maintenance work.

2. All components common to primary coolant systems of LWRs should be included unless specifically precluded by innovative technology applications.

3. Capital costs should be minimized through the use of known and proven technologies and materials.

4. The design and layout of the RPV and internals must facilitate natural circulation flow to allow for passive safety system implementation.

5. Each component of the RPV must be capable of instrumentation and controls for simple operation and monitoring of the reactor coolant system.

Through integration and application of these design criteria, an integral RPV was developed to house the primary coolant system and facilitate economical, safe, and effective operation of the I'S-LWR. The following sections describe the particular design methodologies, parameters, dimensions and schematics of the $I^{2} S$-LWR systems and components.

III. Integral Reactor Pressure Vessel

The most significant deviation from standard LWRs for integral reactors is the design of an effective integral reactor vessel. Unlike standard 2-4 loop LWRs, integral LWRs have effectively zeros loops; the entire primary system is contained within the RPV. The size of the RPV, however is constrained, in that it must not exceed reliable fabrication sizes in order to mitigate capital costs. Currently the EPR-1400 vessel forgings represent one of the largest nuclear forgings to date ${ }^{6}$. At nearly $5 \mathrm{~m}$ in diameter, this represents the upper limit on the reactor pressure vessel size, and this limit is applied to the $\mathrm{I}^{2} \mathrm{~S}$-LWR RPV design. A small sized light water reactor, such as an SMR, can easily satisfy this requirement without sacrificing safety or requiring further design development ${ }^{2}$. Meeting the RPV size requirement for a larger-sized (>1000 MWe) nuclear power plant, however, is significantly more challenging. 


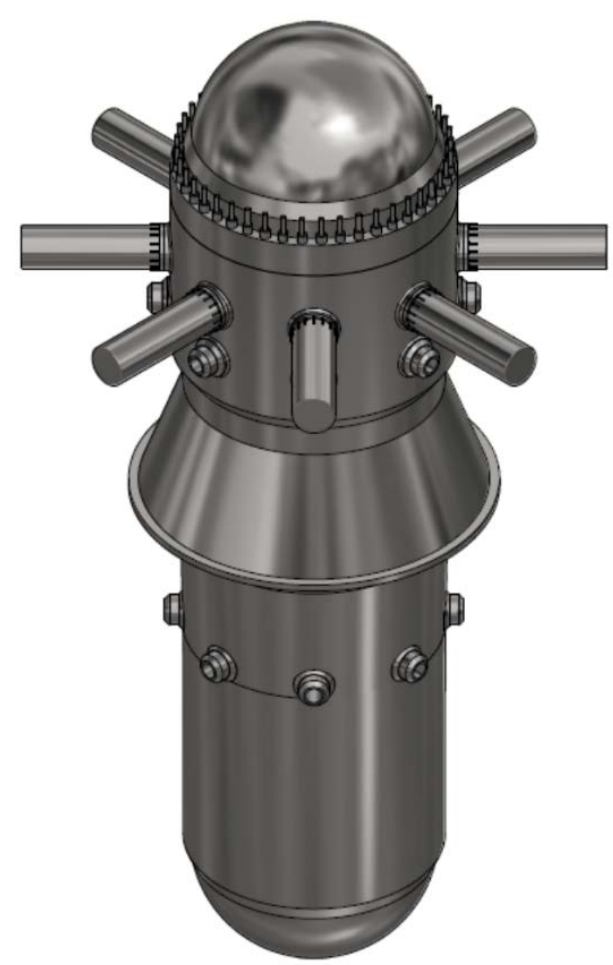

Figure 1: Integral RPV of the I'S-LWR

The RPV is the most complex component as it interfaces and encapsulates each other component. All five of the major design requirements must be considered extensively in the design of the RPV. First, the RPV must be small enough to fall within the forging limitations, but also sufficiently large enough to house the pressurizer, reactor coolant pump hydraulics, instrumentation and controls plus cabling, flow paths, primary heat exchangers, traditional reactor vessel internals, core and control rod drive mechanism (CRDM) support plates, and the reactor core. Additionally, there must be sufficient penetrations for cabling, steam generation system pipes, emergency cooling loop piping, and monitoring systems. Essentially, the RPV cannot be configured as a single component in an integral reactor, and must be developed based upon each of the design constraints and the interfaces with the other primary coolant system components. The integral RPV used in the I'S-LWR concept is similar to those used in other integral reactor designs, with a design pressure and temperature of 2500 psi and $750{ }^{\circ} \mathrm{F}$, respectively. Figure 1 is an illustration of the integral RPV concept developed by the $I^{2} S-L W R$ design team. Each of the primary cooling system components in a traditional LWR was integrated into the concept, with careful focus being placed upon the major design criteria cited in section II. The following sections of this paper describe each of these components, including the design criteria that impacted their development.

\section{III.a. Reactor Coolant Pumps}

The primary coolant system is responsible for transferring core heat to the secondary fluid in the primary heat exchanger within the reactor pressure vessel (RPV). This is facilitated through the reactor coolant pumps (RCP). In conventional LWRs, the reactor coolant pumps are located external to the vessel along the hot or cold legs of the primary coolant system. In the $I^{2} S-L W R$, the fluid path must be integral to the vessel, but considering the first design criteria from section II, there is insufficient space to locate these pumps completely within the reactor vessel. Alternatively, developing an innovative, 
first of a kind, more compact, and thus unproven pump would violate design criteria three listed in section II.

Thus, the I $^{2}$ S-LWR RCPs were designed to attach to the reactor vessel such that the hydraulics are integral to the vessel, while the motor is external to the vessel. Since these pumps are seal-less pumps, the pump casing is transformed into the pressure boundary for the reactor, as is done in the AP1000 reactor ${ }^{4}$. Additionally, the reactor coolant pump motors were oriented radially in order to facilitate sufficient space within the RPV for standard rotational operation of the pump impeller while avoiding the manufacturing difficulties associated with the elimination of portions of the pressurizer hemisphere that would result from orienting the RCP motors vertically.

Pump design methodologies in the power industry are highly proprietary and thus assumption validation is difficult. Simplistic pump design methodologies were used here to scope an appropriate size for the motors and hydraulics of the I $^{2} \mathrm{~S}-\mathrm{LWR} \mathrm{RCPs}^{6}$. The impeller diameter and the horsepower required form the basis for developing the hydraulics and motor, respectively. In sizing these pumps, it is important to consider two major constraints. First, because of the orientation of the RCPs, a centrifugal pump is the design of choice, and thus the specific pump speed, $n_{s}$, must be less than 1.5 , as values of $n_{s}$ greater than this are better suited to mixed or axial flow pumps. Second, the tip speed of the pump impeller must be less than $1700 \mathrm{~m} / \mathrm{s}$, so as to prevent mechanical failure of the impeller components.

The impeller diameter is used to determine RPV penetration size, and thus is the first design constraint to be developed in sizing the RCPs. The impeller diameter is can be found by ${ }^{6}$ :

$$
D=\frac{2 \phi \sqrt{2 g H}}{\omega}
$$

Where:

$$
\begin{aligned}
& D=\text { the impeller diameter }(\mathrm{in}) \\
& \omega=\text { the angular velocity }(\mathrm{rpm}) \\
& \mathrm{g}=\text { the gravitational constant }\left(\mathrm{ft} / \mathrm{s}^{2}\right) \\
& \mathrm{H}=\text { the head }(\mathrm{ft}) \\
& \Phi=\text { the overall head coefficient. }
\end{aligned}
$$

The head is based on the coolant flow rate and flow path throughout the primary coolant system. For normal operation of the $I^{2} S-L W R$, the best estimated flow rate is $254,028 \mathrm{gpm}$ with a corresponding pressure drop of $482.2 \mathrm{kPa}$. The overall head coefficient was obtained based on these parameters from a canonized plot in the reference text ${ }^{4}$. A plot of the impeller diameter as a function of the angular speed of the pumps is given in Figure 2. 


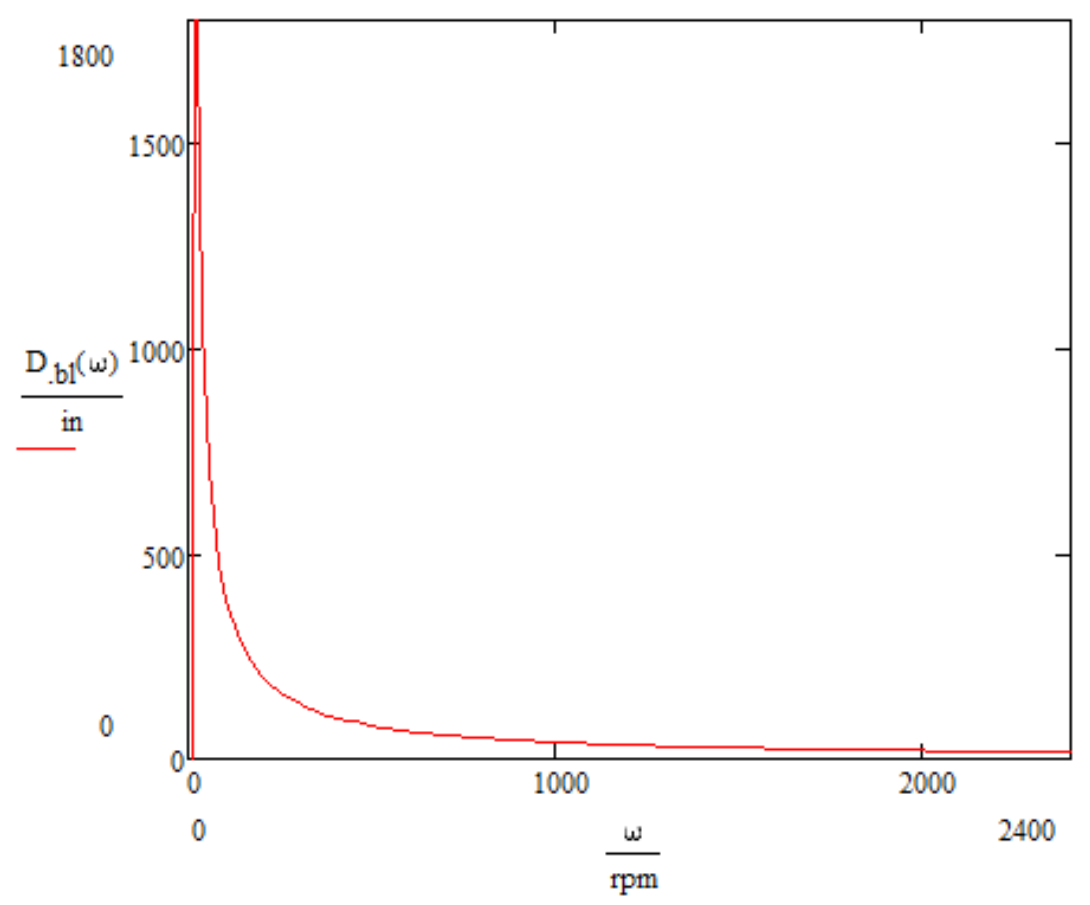

Figure 2: Hydraulic diameter in inches as a function of the angualr speed in rpm for the I'S-LWR RCPS

The impeller diameter is independent of flow rate, but the brake horsepower, and thus motor size, is directly impacted by the flow rate through each pump. As the pumps are in a parallel arrangement, the head across each pump is equal to the total pressure drop in the reactor pressure vessel fluid flow path. However, the flow through each pump is divided by the number of pumps. Hence, flow rate and the number of pumps are integral factors in determining the brake horsepower. The fluid horsepower, FHP, is determined using:

$$
F H P=\dot{m} g H,
$$

where $\dot{m}$ is the mass flow rate $(\mathrm{lb} / \mathrm{s})$. The brake horsepower, BHP, can be found considering the mechanical efficiency of the pump, $\eta$, using:

$$
B H P=\frac{F H P}{\eta}
$$

The efficiency for centrifugal pumps is well established, and can be determined based upon the $\mathrm{n}_{\mathrm{s}}$ from any conventional fluid mechanics textbook ${ }^{7}$. However, in order to determine the pump efficiency, the pump type and pump specific speed should be determined. As mentioned earlier, centrifugal pumps are the best fit for the $I^{2} S$-LWR RCPS. The pump specific speed is a dimensionless number used to characterize the performance of a pump at its optimum conditions, and is a common metric determined in initial pump sizing. This value can be determined from:

$$
n_{s}=\frac{\omega Q^{05}}{(g H)^{0.75}}
$$

Where $Q$ is the volumetric flow rate through each pump. 
The pump specific speed can be used to determine the ideal pump type to be used in a given application. As the pump of choice for the $I^{2} S-L W R$ is a centrifugal pump, $\mathrm{n}_{\mathrm{s}}$ must be less than 1.5 , which is the range of specific speed for which centrifugal pumps are utilized. The efficiency range for these centrifugal pump is from approximately $70 \%$ to $92 \%$. As the specific speed of a pump depends on the flow rate through the pump, the number of pumps directly impacts the specific speed. This is best illustrated in Figure 3, where the limiting specific speed is depicted as a horizontal dashed line. The number of pumps also impacts the structural makeup of the RPV, since additional penetrations at the same elevation decrease the structural integrity of the vessel. Based on the calculations described here, the optimal number of parallel pumps for this integral vessel was found to be eight.

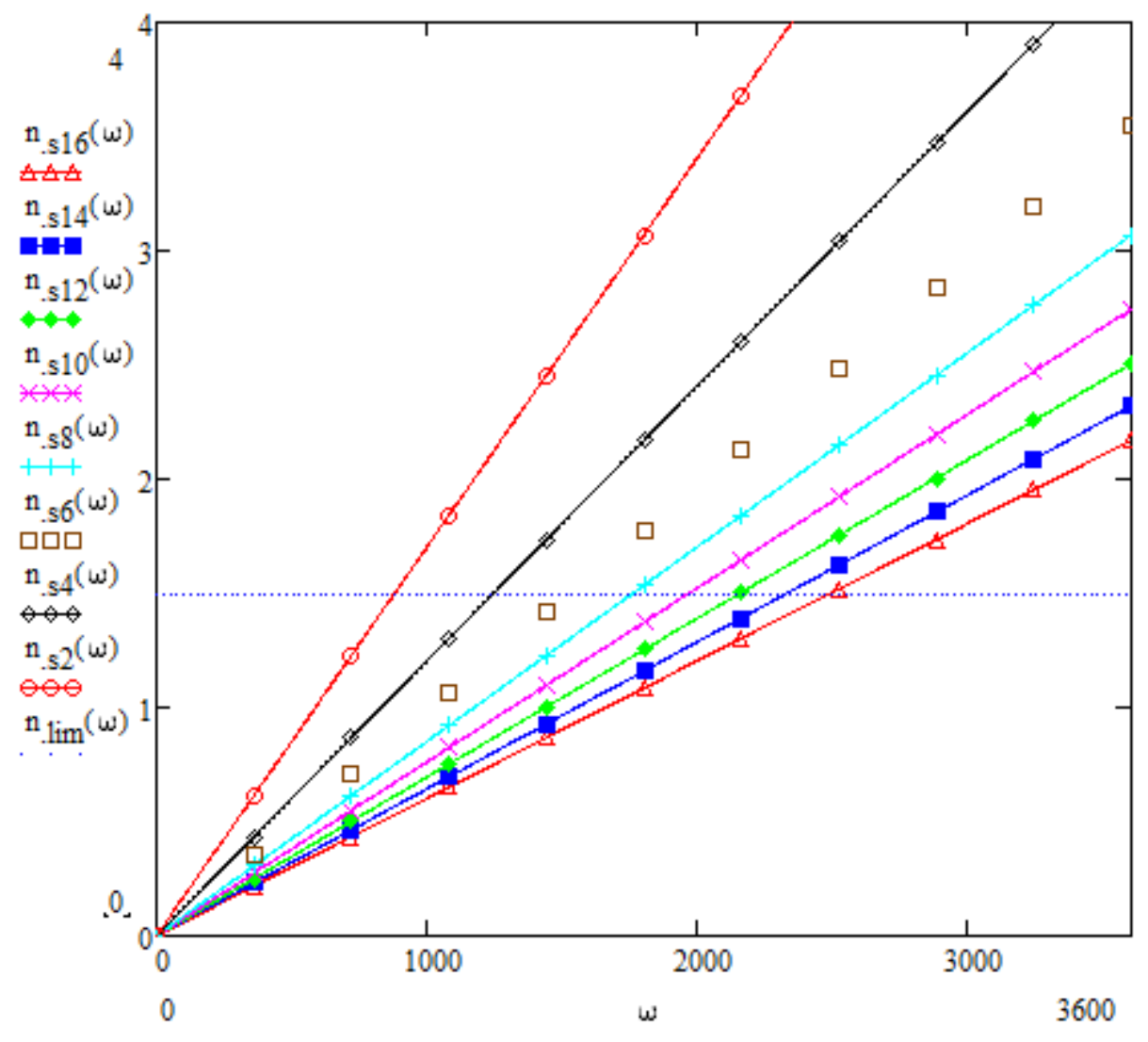

Figure 3: Specific speed of the I'S-LWR RCPs given 2, 4, 6, 8, 10,12, 14, or 16 pumps in parallel.

Once the brake horsepower and impeller diameter were found, the pump motor type and size were determined. The frame best suited to accommodate the requirements of the I'S-LWR RCPs is an E7111 sized motor $^{8}$. Based on this metric, the length, diameter and weight of the pumps were estimated. These estimates were a pump length of $110 \mathrm{in} ., 40 \mathrm{in}$. for the motor diameter, and $13000 \mathrm{lbs}$. for the weight. The RPC parameters developed from this study for several pump speeds can be found in Table 1. The speed and corresponding pump parameters corresponding to the selected based pump design are highlighted in yellow. 
Table 1: I'S-LWR RCP potential and base (yellow) design parameters

\begin{tabular}{|l|l|l|l|l|}
\hline Pump Speed (RPM) & 2400 & 1800 & 1400 & 900 \\
\hline Impeller Diameter (in) & 16.2 & 21.6 & 28.9 & 43 \\
\hline Specific Speed & 2.2 & 1.6 & 1.2 & 0.818 \\
\hline Efficiency (\%) & 84.5 & 85.5 & 87.0 & 89.5 \\
\hline Motor Power (HP) & 3507 & 3466 & 3406 & 3311 \\
\hline Motor Length (in) & 110 & 110 & 110 & 110 \\
\hline
\end{tabular}

The location of the RCPs with respect to the RPV depends upon spatial and mechanical stress considerations. In order to provide sufficient room for the impeller, the pump must be located at the top of the RPV (above the primary heat exchangers) or at the bottom of the RPV. Therefore, a stress analysis was completed for both of these potential configurations to determine the most likely location for the pumps.

The steel used to determine the stresses was ATSM designated A543 B Class2 Low alloy steel. This was chosen for the pressure vessel steel based on a frequently referenced paper written by R. H. Sterne and L. E. Steele ${ }^{9}$. The authors of this paper discuss the different steels that have been used in RPVs up until that point, and from their findings the steel stated above was chosen as an ideal template to predict stresses and failures for the I'S-LWR RPV. The data used in the analysis of the pressure vessel was obtained from the ASTM material data sheets which are readily available online ${ }^{10}$.

The basic stress equations ${ }^{11}$ were used to evaluate the stresses in I $^{2}$ S-LWR RPV:

$$
\begin{gathered}
\sigma_{t}=\frac{\mathrm{r}_{i}^{2} \mathrm{P}_{\mathrm{i}}}{r_{o}^{2}-r_{i}^{2}}\left(1+\frac{\mathrm{r}_{\mathrm{o}}^{2}}{r^{2}}\right) \\
\sigma_{r}=\frac{\mathrm{r}_{\mathrm{i}}^{2} \mathrm{P}_{\mathrm{i}}}{r_{o}^{2}-r_{i}^{2}}\left(1-\frac{\mathrm{r}_{o}^{2}}{r^{2}}\right) \\
\sigma_{n}=\frac{M c}{I}
\end{gathered}
$$

Where:

$\sigma_{\mathrm{t}}=$ the tangential stress

$\sigma_{\mathrm{r}}=$ the radial stress

$\sigma_{\mathrm{n}}=$ the normal stress

$\mathrm{P}=$ the internal pressure

$r_{0}=$ the outer radius of a cylinder

$r_{i}=$ the inside radius of a cylinder

$r=$ the radius at which the stress is evaluated

$I=$ the wall moment of area

$\mathrm{M}_{\mathrm{c}}=$ the bending moment. 
Because of the difficulties that arise from having a non-uniform thickness in the pressure vessel in addition to the challenges of placing holes in the same pressure vessel, attaching a counterweight, such as a radially oriented reactor coolant pump could substantially challenge the vessel. These simplistic equations, though valid in principle, cannot effectively be used to calculate the stresses on the vessel due to reactor coolant pump mounting. Thus, a detailed mechanical stress model was developed to analyze the impact of pressure and mechanical stresses on the RPV wall.

The computer code ANSYS was used to perform a finite element analysis on the vessel wall. The RPV was first created in Autodesk Inventor 2014 and then imported into ANSYS where appropriate operational loads and constraints were applied. Because of the effective RPV symmetry, only oneeighth of the pressure vessel was modeled and symmetric boundary conditions were utilized. As part of the stress analysis in ANSYS, the following assumptions were made:

1. The pump is cantilevered onto the pressure vessel.

2. The entire weight of the pump is conservatively located at the end of the pump rather than the center.

3. The pressure inside the vessel was the same as the pressure inside each of the RCPs, and this pressure was the maximum design pressure.

Based on these assumptions and the design parameters of the RPV and RCP, the developed stress maps are illustrated in Figure 4 through Figure 6.

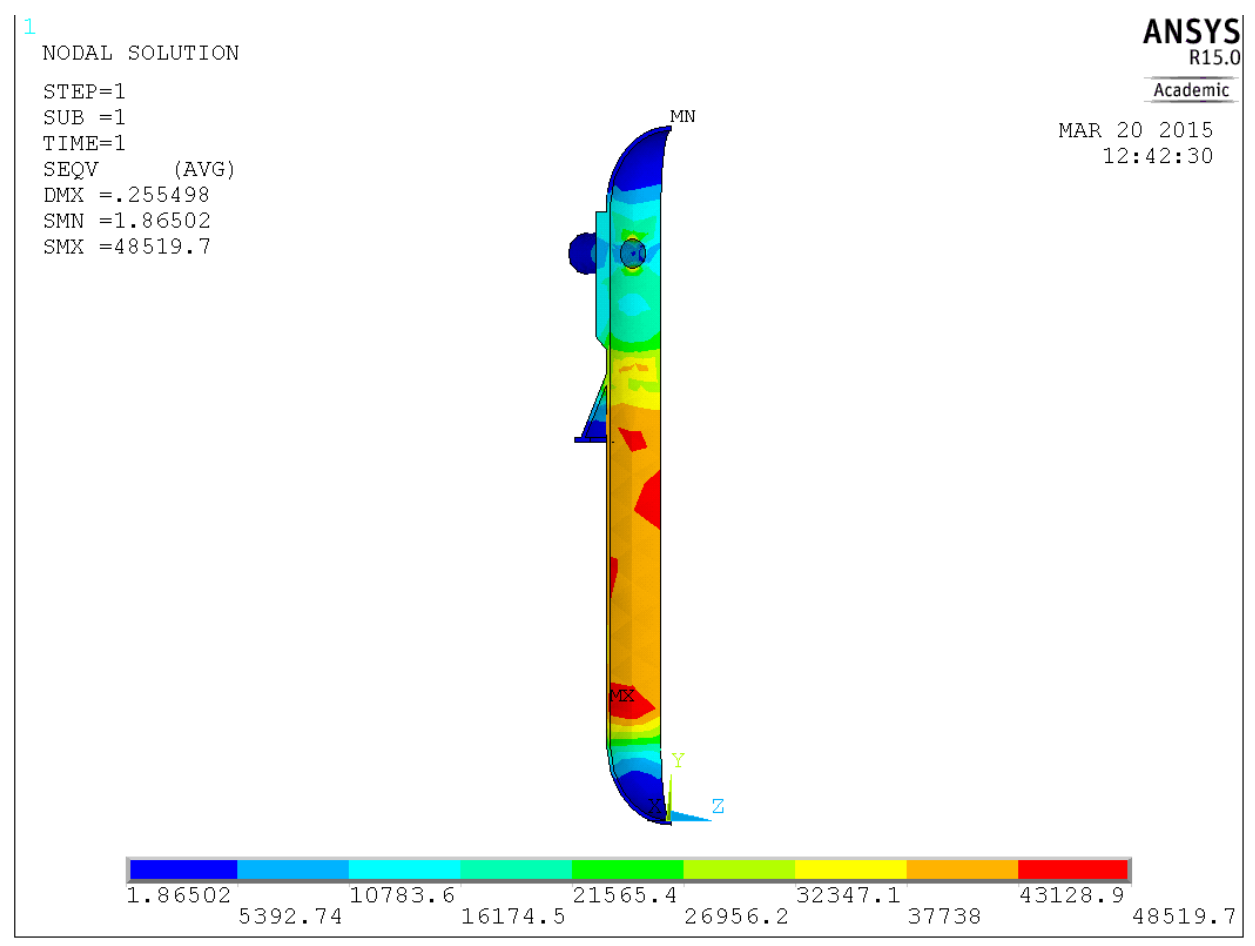

Figure 4: Von Mises Stresses of the RPV wall (psi) for top mounted RCPs

As can be seen from Figure 4, the maximum stress RPV with top mounted RCPs is the lower end of the reactor vessel, which is several meters from the connection with the pump. These stresses are due to the pressure inside of the vessel, which affects the thinner-walled region of the RPV. The total deflection in all directions as a result of the stresses is plotted in Figure 5. 


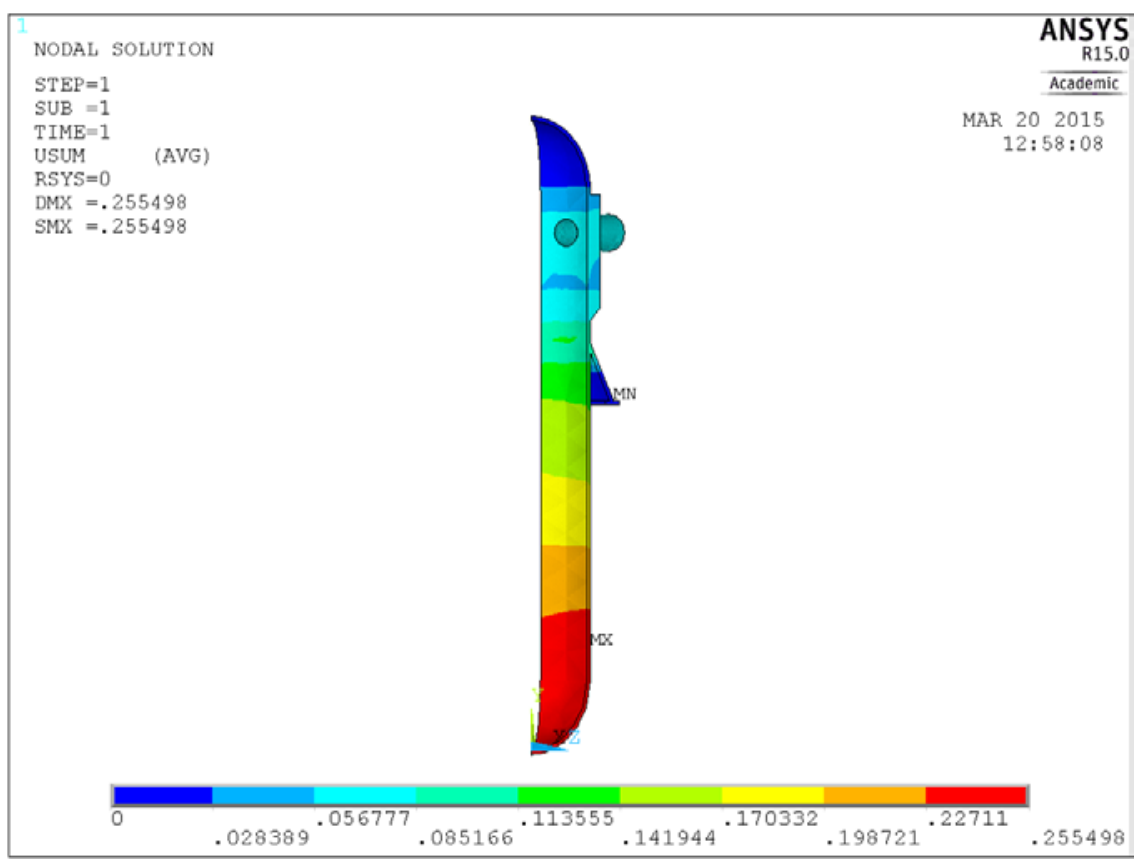

Figure 5: Total wall deflection of the RPV wall (inches) in all directions for top mounted RCPs When the pumps were next located near the bottom of the vessel, stresses were analyzed in ANSYS using similar assumptions as for the top mounted RPCs. Figure 6 illustrates the stress map for bottommounted RCPs.

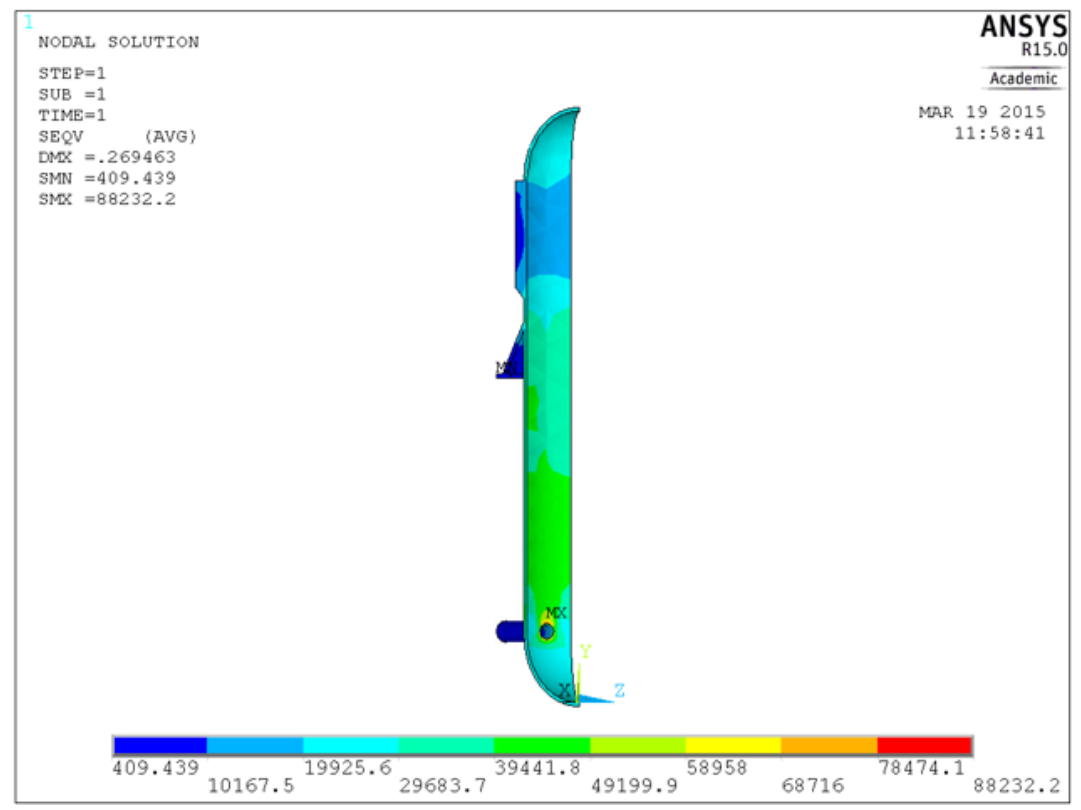

Figure 6: Von Mises Stresses of the RPV wall (psi) for bottom mounted RCPs.

These results indicate that mounting the reactor coolant pumps at the bottom of the pressure vessel wall results in vessel stresses nearly doubled those developed when the same pump is mounted at the 
top of the reactor vessel. This is primarily due to the increased structural integrity derived from the increased thickness of the RPV at the top of the vessel.

To ensure that the RPV will not fail under normal operating conditions, a rough estimate of the safety factor for the stresses that can be allotted to this vessel was made. The equation for the safety factor is:

$$
\eta=\frac{s_{y} \sqrt{2}}{\sqrt{\left(\sigma_{1}-\sigma_{2}\right)^{2}+\left(\sigma_{2}-\sigma_{3}\right)^{2}+\left(\sigma_{3}-\sigma_{1}\right)^{2}}}
$$

Where:

$$
\begin{aligned}
& \text { Sy }=\text { the yield stress } \\
& \eta=\text { the radial stress } \\
& \sigma_{1,2,3}=\text { the directional components of the stress }
\end{aligned}
$$

The safety factor calculated for the vessel with top mounted pumps was 2.06 , while the safety factor for the bottom mounted pumps was 1.14. The safety factor associated with the bottom mounted pumps is quite small, and is far below the desired design value of 2.0. Therefore, the base design of the I'S-LWR RPV includes top mounted reactor coolant pumps with parameters established in Table 2.

In order to validate and verify the ANSYS results, hand calculations were performed to approximate the stresses at two points in the vessel walls to validate the model results. The same assumptions and parameters were used for these calculations. The connection of the pressure vessel to the pump wall at the top outside section of the RPV was selected as the first point for stress calculation. The other point that was chosen was a point in the vessel wall of the cylindrical RPV section below the skirt. The stress for the top portion of the pump cantilevered onto the pressure vessel was determined to be $10275 \mathrm{psi}$. The pressure on the outside of the vessel in between the skirt and the thickened section was determined to be 20231psi. These results indicate similar trends and reasonably similar scale as the modeled results, indicating reasonable conclusions based upon the ANSYS model.

The final results of the pump analysis provide general size and parameter information of pumps required to pump primary coolant through the $I^{2} S$-LWR primary system. Although base design and location selections were made, further analysis will be need to be done in order to evaluate detailed pump internals design and vibration analysis with respect to the RPV stress loads.

Table 2: Base Design Parameters for the I2S-LWR top-mounted RCP

\begin{tabular}{|l|c|}
\hline \multicolumn{1}{|c|}{ Design Parameter } & Value \\
\hline Number of Pumps & 8 \\
\hline Rotational Speed (RPM) & 1400 \\
\hline Pressure Drop (psi) & 115.2 \\
\hline Water Specific Gravity & 0.728 \\
\hline Total Flow Rate (gpm) & 349309 \\
\hline Pump Efficiency (\%) & 87 \\
\hline Frame Size ${ }^{8}$ & E7111 \\
\hline Length (in) & 110 \\
\hline Impeller Diameter (in) & 27.7 \\
\hline
\end{tabular}




\section{III.b Pressurizer}

The pressurizer (PZR) is a critical component for pressurized water reactors (PWRs), since it performs two fundamental functions. First, it provides the means for monitoring and controlling the reactor coolant system pressure to ensure that a sufficient degree of sub-cooling in the core is maintained during plant operation. Second, it provides the means of monitoring the reactor coolant system inventory, which is necessary to preclude the potential for a dangerous reduction in the liquid level in the system.

The initial pressurizer design for the $I^{2} S-L W R$ is shown in Figure 7. The pressurizer is located in the upper head of the reactor pressure vessel, above the internal control rod mechanisms. This layout is considered optimal for the $\mathrm{I}^{2} \mathrm{~S}$-LWR design, operating with both of the closure flanges at a uniform temperature, and maximizes the overall pressurizer volume, while providing adequate space for placement of the reactor coolant pumps and internal control rod drive mechanism (CRDMs) [12, 13].

In the present design, the total height of the PZR is $4.97 \mathrm{~m}$ and the total volume is $54.62 \mathrm{~m}^{3}$. The top part of the PZR consists of a hemisphere with radius $2.35 \mathrm{~m}$, while the lower part consists of a $1.32 \mathrm{~m}$ high cylindrical sector. At the bottom of the PZR volume, a $1.3 \mathrm{~m}$ high surge section with orifices is present. The nominal water level is allocated between the intermediate cylindrical section and the hemispherical region. The surge orifices are designed to connect the PZR to the reactor primary circuit, and take over the function of the surge line of a typical PWR. Thermal insulation is used to minimize heat losses from the PZR into the primary circuit (see Figure 7). At nominal conditions, the water level is about $3.25 \mathrm{~m}$, corresponding to a water volume of about $42.5 \mathrm{~m}$.

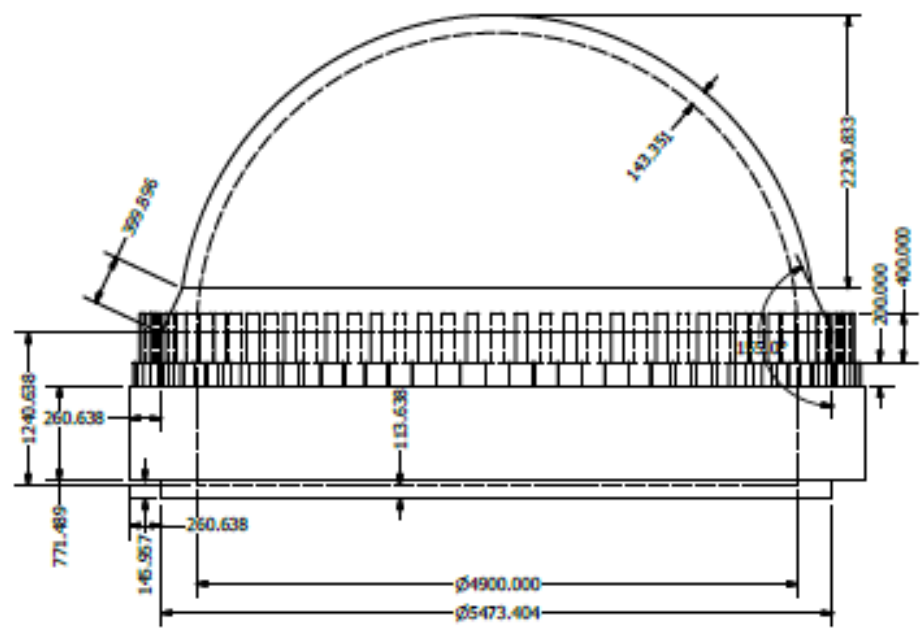

Figure 7: Pressurizer design configuration for the I'S-LWR concept 

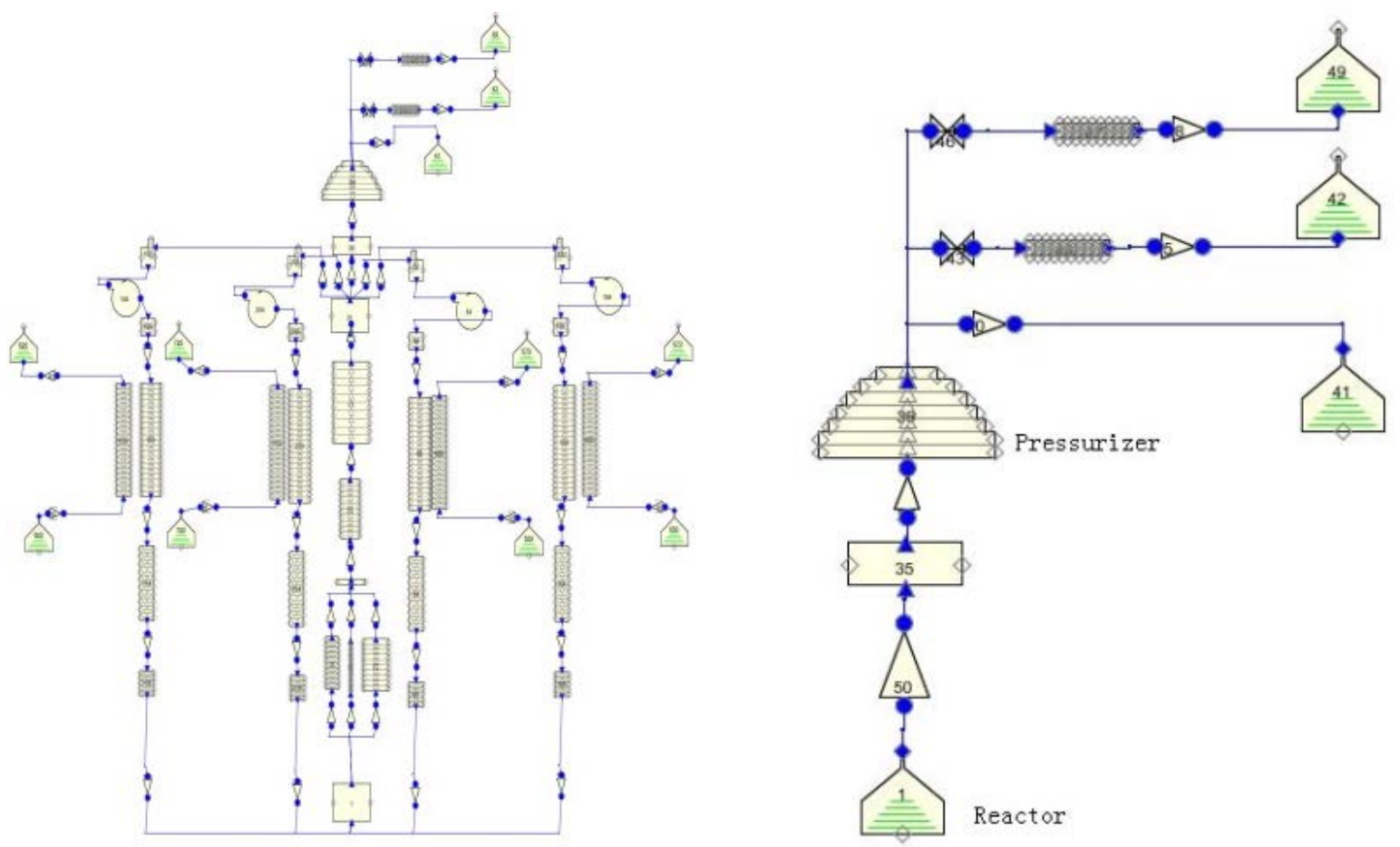

Figure 8: I'S-LWR reactor vessel and Pressurizer RELAP5 nodalizations

In order to perform a first assessment of the design of the I $^{2} S$-LWR PZR and optimize the system dimensions, a stand-alone RELAP5 model for the PZR system was developed. The RELAP5 nodalization, shown in Figure 2 , included a sufficiently detailed model of the $I^{2} S$-LWR primary system to allow an acceptable estimation of the surge flow and temperature during various operational maneuvers. The PZR thermal hydraulic characteristics were then investigated under different combinations of reactor coolant temperature/power and pressurizer water volume/level conditions. The simulation results are presented in Figure 9 through Figure 11.

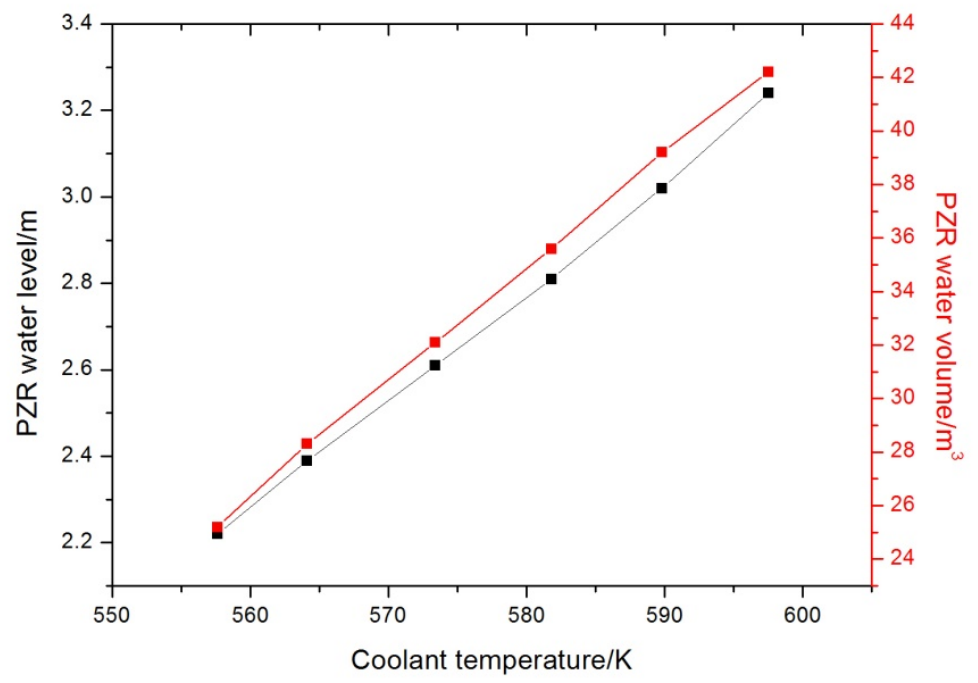


Figure 9: Variations of PZR water level and water volume vs coolant temperature

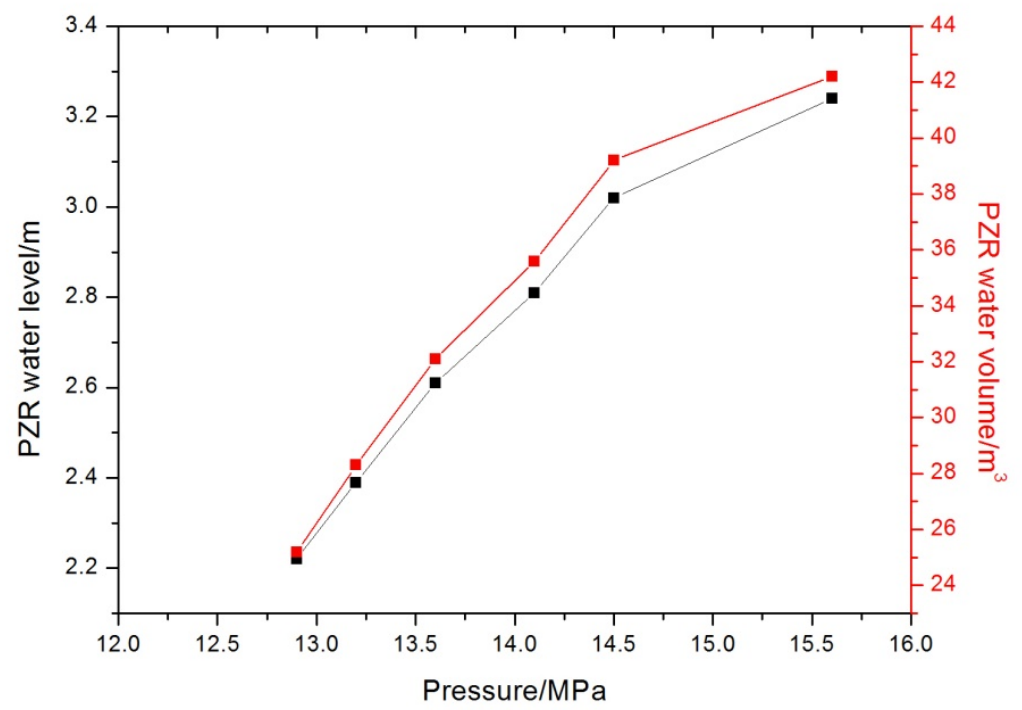

Figure 10: Variations of PZR water level and water volume vs pressure

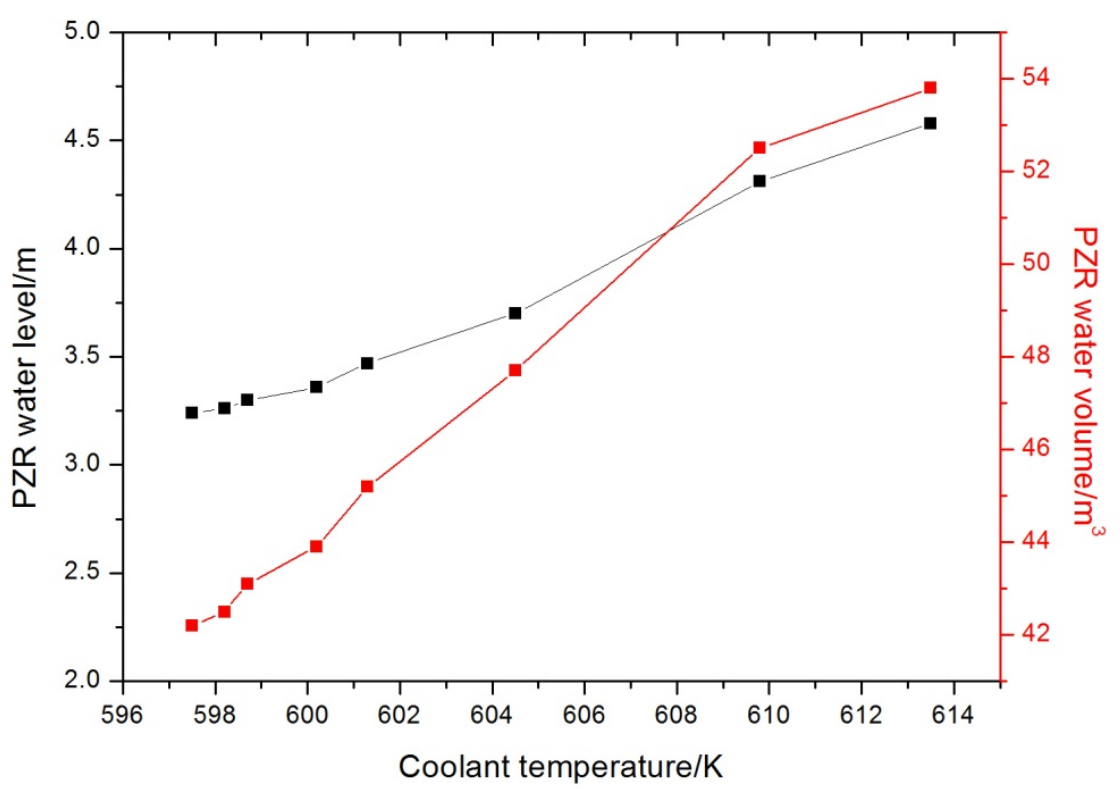

Figure 11: Variations of PZR water level and water volume vs coolant temperature due to a decreasing MCHX secondary flow 
The reactor core power is varied from $3000 \mathrm{MW}$ to $300 \mathrm{MW}$, while the coolant temperature is decreased from $598 \mathrm{~K}$ to $557 \mathrm{~K}$. Under these conditions, the pressure decreases from $15.6 \mathrm{MPa}$ to 12.9MPa. The corresponding PZR water volume and PZR water level as a function of coolant temperature and system pressure are reported in Figure 9 and Figure 10 respectively. In a second set of simulations, a decrease of the microchannel heat exchanger (MCHX) secondary side flow is tested, which leads to an increase of the primary coolant temperature from $598 \mathrm{~K}$ to $614 \mathrm{~K}$. The variations of PZR water volume and water level with coolant temperature for this case is shown in Figure 11. The results demonstrate that the water level in the PZR can be maintained at acceptable values over a wide operational range. The transient behavior of the pressurizer during selected accident scenarios is demonstrated in two companion papers [14, 15]. A drawing of the pressurizer concept can be found in Figure 12.

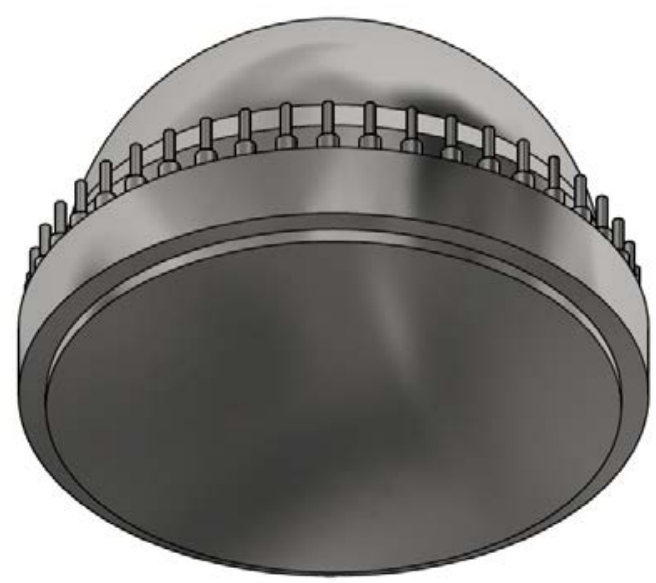

Figure 12: Drawing of the I'S-LWR Pressurizer

\section{III.c Primary Heat Exchangers}

The primary heat exchangers transfer heat from the hot primary coolant to the steam generation system (SGS) working fluid. Unlike Gen III and earlier reactors, which use large U-tube steam generators, the heat exchangers in the $I^{2} S$-LWR primary system must be compact and robust. Thus, a microchannel heat exchanger (MCHX) concept was developed, which is capable of transferring large amounts of heat using significantly less volume than conventional shell and tube heat exchangers. In these exchangers, flat $1.016 \mathrm{~mm}$ metal plates are etched with $0.635 \mathrm{~mm}$ semicircular channels. These plates are stacked on top of one another, with each plate being rotated azimuthally 90 degrees relative to the plate below it. This facilitates two separate flow paths, one for hot fluid and one for cold fluid, as illustrated in Figure 13. Additionally, the cold plates include two 90 degree bends, which allow the MCHX to operate in a counter-current configuration. 


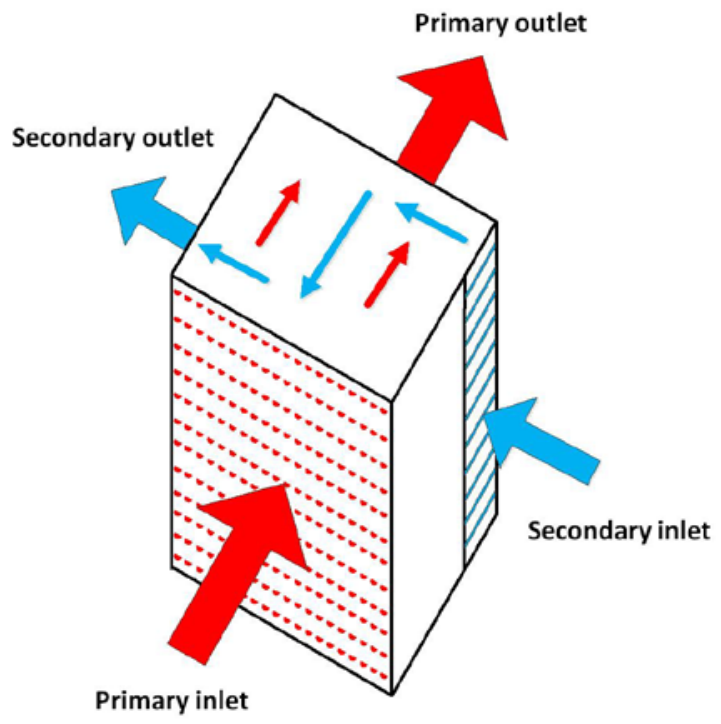

Figure 13: Illustration of flow paths in the microchannel heat exchanger concept

The overall I'S-LWR MCHX design consists of 420 channels per plate with 590 plates stacked into a single unit. 11 units are stacked into a heat exchanger column and eight columns are included in the annular downcomer region of the RPV. The primary fluid, once ejected from the pump hydraulics, flows downward through the annular region of the RPV, and into a rectangular header that runs the length of the header stack. The width of this header decreases as the stack elevation increases, increasing the hydraulic resistance along the length of the header. This ensures that a similar flow rate is achieved through each of the microchannels along the stack height. The cold fluid for the $\mathrm{MCHX}$ is drawn from the steam generation system, as described in a companion paper to this volume $^{16}$, enters through a piping penetration in the RPV at the bottom of the stack, which then flows into a header on the outside edge of a stack. Cold water then traverses the MCHX channels, and upon being heated, enters a header on the inside edge of the stack, flowing upwards through piping and out of the RPV through a penetration near the top of the MCHX stack. Each of the four steam generation loops feeds into 2 stacks of MCHX units, as illustrated in Figure 14. The design, performance, and validation of these exchangers is described in an accompanying paper ${ }^{17}$.

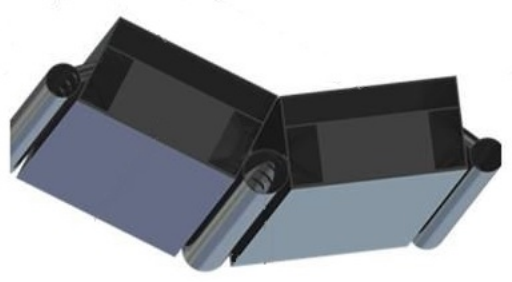

Figure 14: Top-down view of heat exchanger stacks with commensurate piping and headers for heat exchange with SGS loops. 


\section{III.d Decay Heat Removal Heat Exchangers}

The decay heat removal exchangers serve as the means of removing decay heat from the reactor core during unexpected transients. They are helical coil shell and tube heat exchangers, in which hot fluid flows up through inlet piping, through the center of the cylindrical exchanger, into a header in the top hemispherical head of the exchanger, then down through a series of concentric helical tubes. Cold fluid from an intermediate loop enters the reactor pressure vessel through RPV penetrations at the bottom of the exchanger, then enters the shell side of the exchanger, effectively exchanging heat with the hot fluid in the helical coil tubes. These exchangers, shown in Figure 15, are described and evaluated in a separate paper $^{18}$.
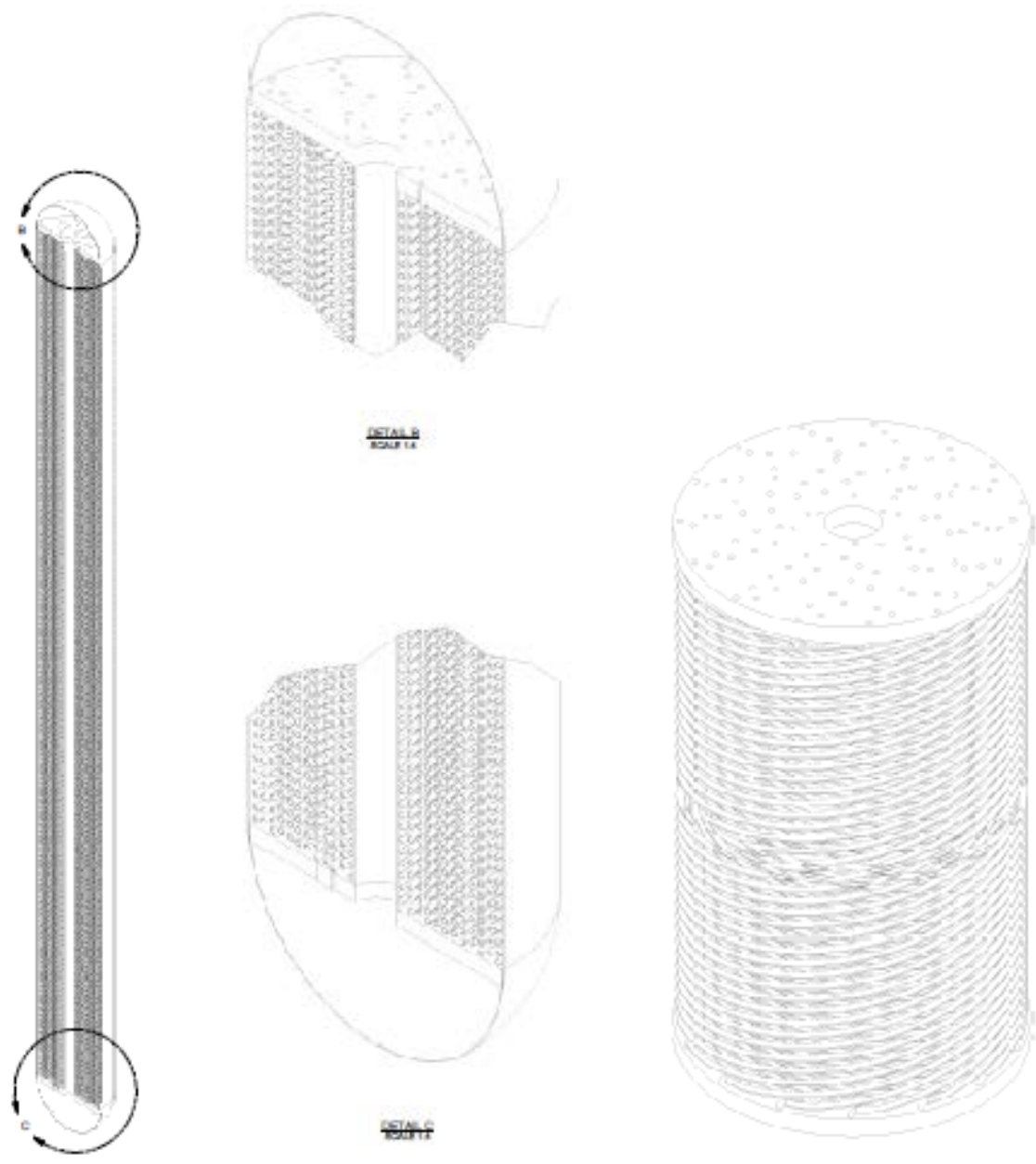

Figure 15: Illustration of the decay heat removal heat exchangers for the $I^{2} S$ - LWR

III.e Reactor Vessel Internals 
The balance of the primary reactor coolant system and RPV design consist of a group of components commonly referred to as the reactor vessel internals. These components provide structural support, flow path modifications, neutron control, and mechanical stability for the primary coolant system and nuclear core. The reactor vessel internals for the $I^{2} S$-LWR include the core support plates, the control rod drive system, the core barrel and other flow components, the neutron reflectors, and the fuel assemblies. The follow sections briefly describe these components and indicate the specifications and design for each of these components.

\section{III.e.i Core Support Plates}

There are two core support plates for the I'S-LWR concept. The role of these plates is three-fold. First, these plates provide mechanical support for the fuel and fuel assemblies. Second, the plates facilitate equally distributed water flow through each of the assemblies, and third, these plates provide pins and grooves for proper alignment and loading of the fuel assemblies and neutron reflectors in the core.

The bottom core plate rests on the core barrel structure, and is designed to withstand the weight of the full nuclear core and core reflectors. There are five holes through this plate at each fuel assembly location to allow for adequate water flow. Additionally, there are two pins that fit into appropriately sized notches in the feet of each fuel assembly. Thus, properly seated assemblies will not slip or adjust position under the large momentum of the incoming water during normal operation. A schematic of the core support plate can be seen in Figure 16.
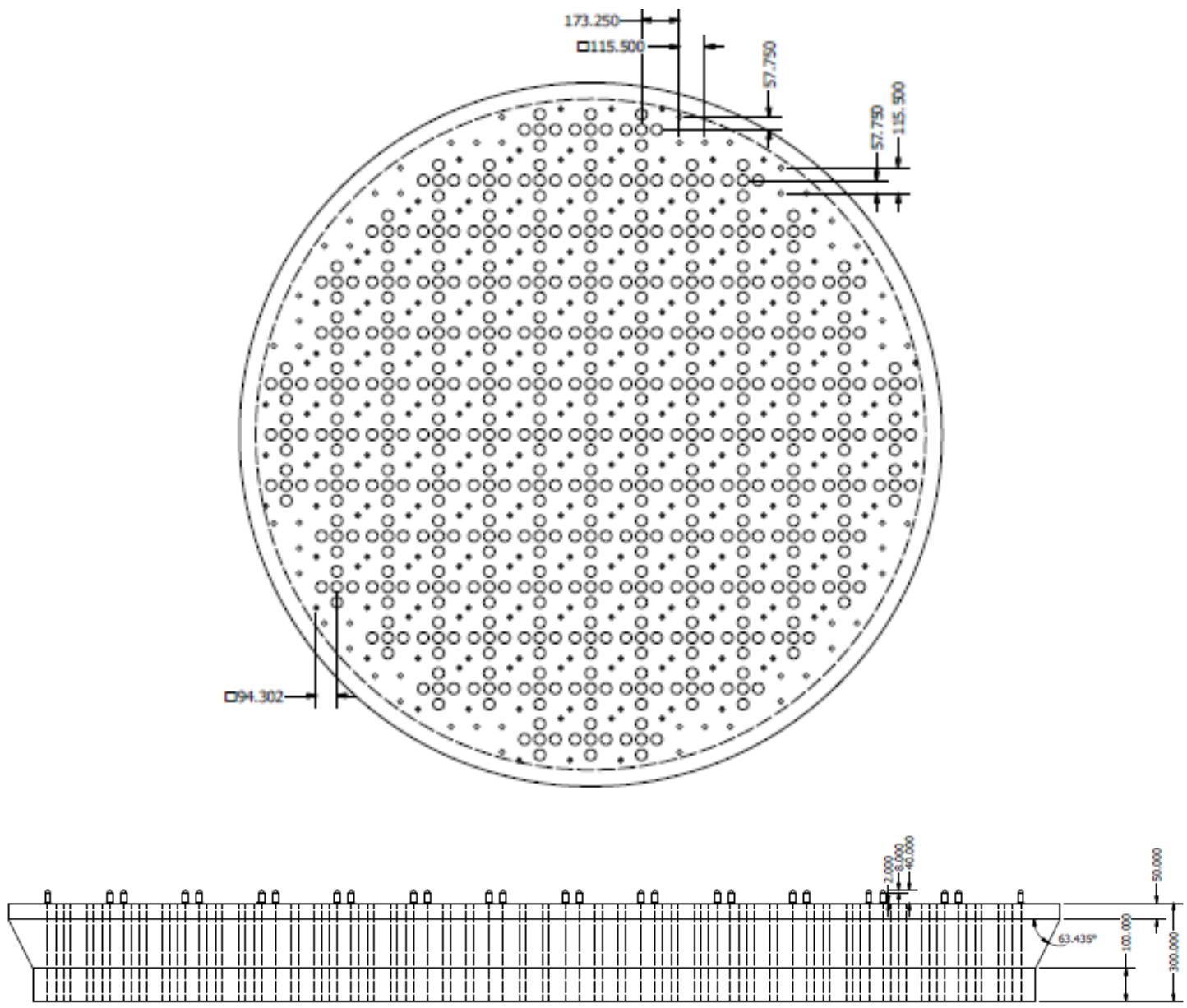
Figure 16: I'S-LWR core support plate schematic

The upper core plate is nearly exactly the same in geometry as the core support plate, except for the support ridge that rests on the bottom of the bore barrel. Both these plates are included as part of the core support system.

\section{III.e.i Fuel Assemblies}

The fuel assemblies are derived from Westinghouse $17 \times 17$ fuel assemblies, modified to account for $19 \times 19 \mathrm{U}_{3} \mathrm{Si}_{2}$ fuel rods. The spacer grids, loading springs, footers and headers, bottom nozzle pads, fluid mixer grids and mixing vane grids are all similar to the $17 \times 17$ fuel assemblies, and are illustrated in Figure 17. Detailed data concerning the core configuration and performance can be found in accompanying papers ${ }^{19,20}$. The overall core configuration, which is also described in accompanying papers, is illustrated in Figure 18.
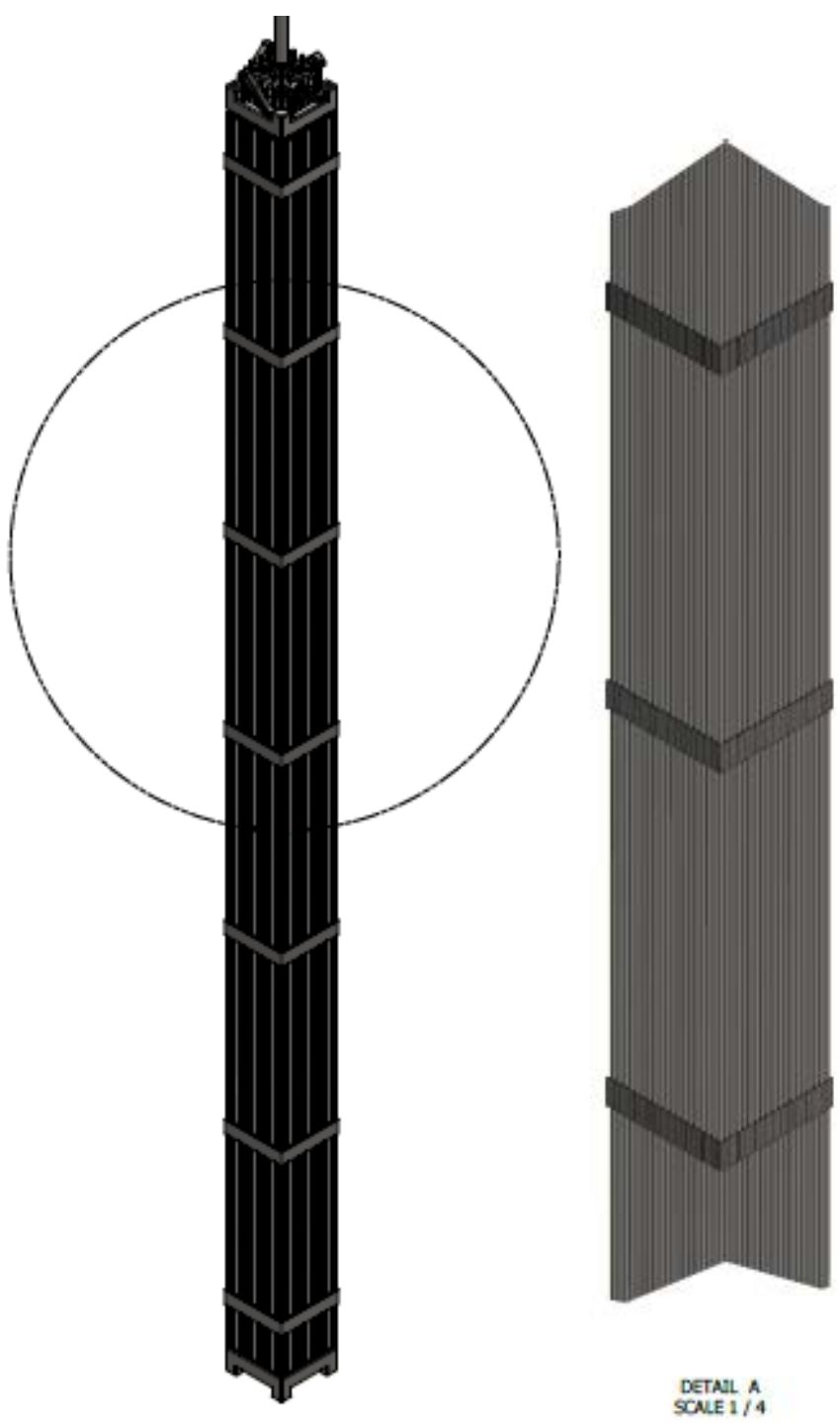

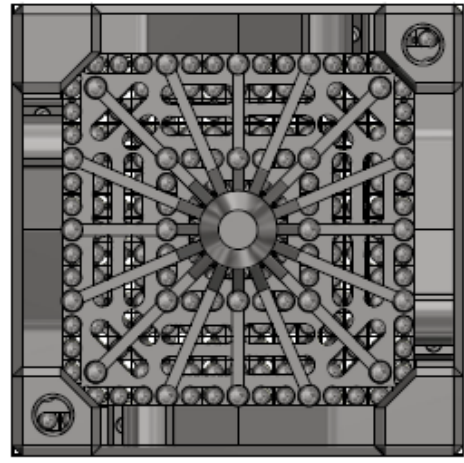

Top View

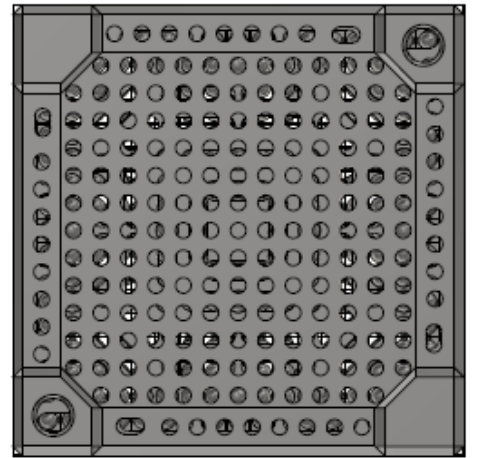

Bottom View 
Figure 17: 19x19 fuel assemblies in the I'S-LWR core

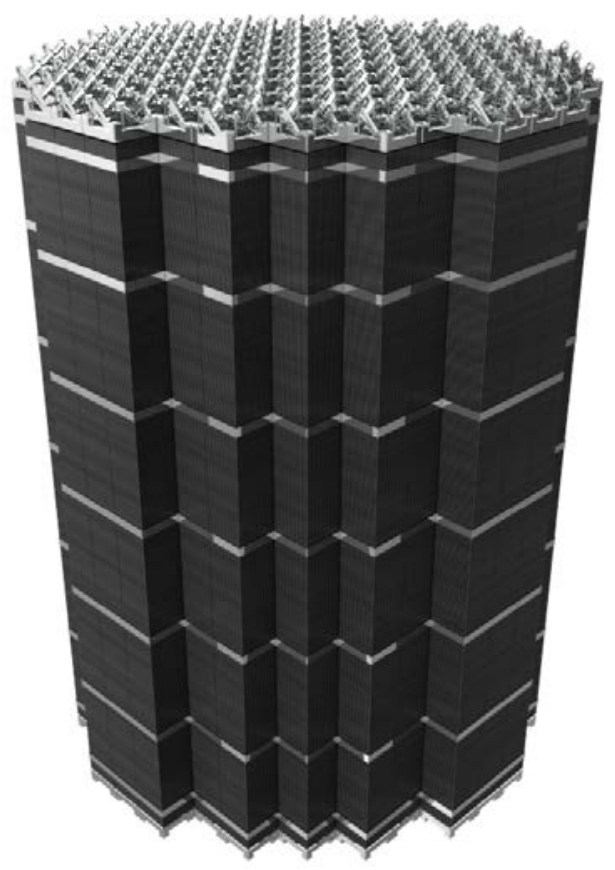

Figure 18: Illustration of the I²S-LWR full core

\section{III.e.i Core Barrel and Flow Path Components}

Instead of providing piping legs to conduct fluid throughout the primary system, the RPV design itself must facilitate coolant flow while minimizing hydraulic losses. To this effect, there are several flowassisting devices in the RPV, including but not limited to rounded corners, gradual diameter changes, expanded flow paths, and metal structures to otherwise induce mixing and direct flow changes while minimizing hydraulic losses.

The flow path in the $I^{2} S-L W R$ is similar to those found in other integral reactor designs, such as the Westinghouse SMR ${ }^{21}$. In the I2S-LWR, the heated fluid from the core rises past the control rod drive system and cabling above the core through the equivalent of the hot leg. The core barrel, illustrated in Figure 18, separates the cold and the hot fluids in the primary system, and also provides support for the core. This hot fluid which flows upward through the core barrel, then hits the pressurizer plate and flows radially through holes in the core barrel into the reactor coolant pump inlet. The pump effluent thus flows down into the annular region of the RPV, and from there into the MCHXs. The MCHX effluent enters the lower annular region of the RPV, which is equivalent to the cold leg of conventional LWRs. Upon reaching the bottom of the RPV, this fluid then turns and is directed upward into the core assemblies through the core support plate. 


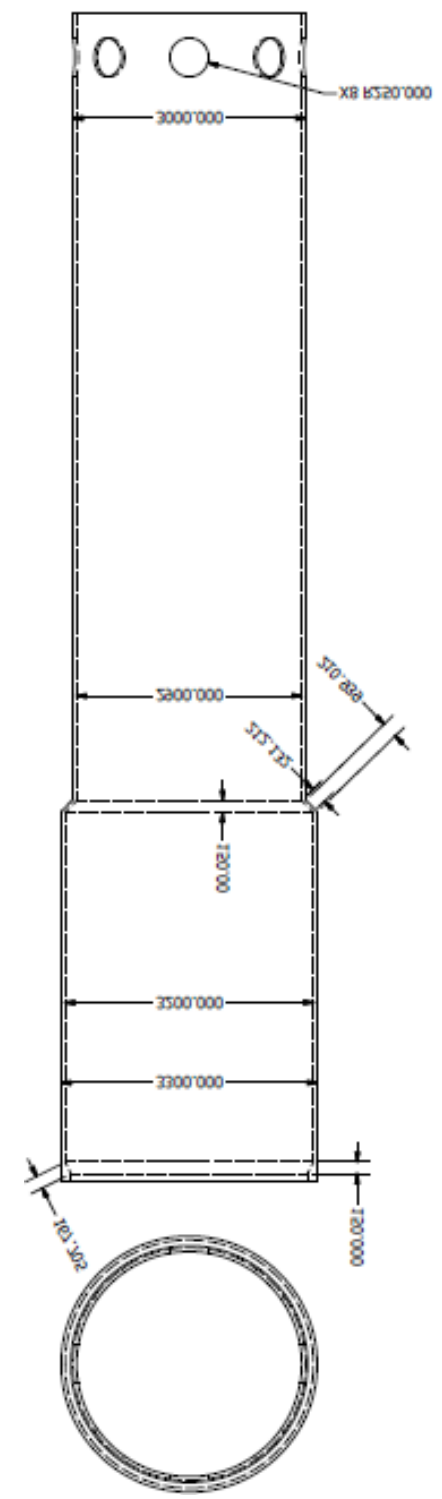

Figure 19: Core barrel of the I2S-LWR RPV

\section{III.e.i Reflectors}

Neutron reflectors are included in the I2S-LWR to decrease radial neutron leakage and improve the overall reactor economy. It is made of several steel rings sections, cut to encircle the core, stacked to the height of the active fuel rods. Small circular holes are drilled through each reflector section in order to facilitate water flow through the reflector to cool the steel. An illustration of the reflector is seen in Figure 20. 


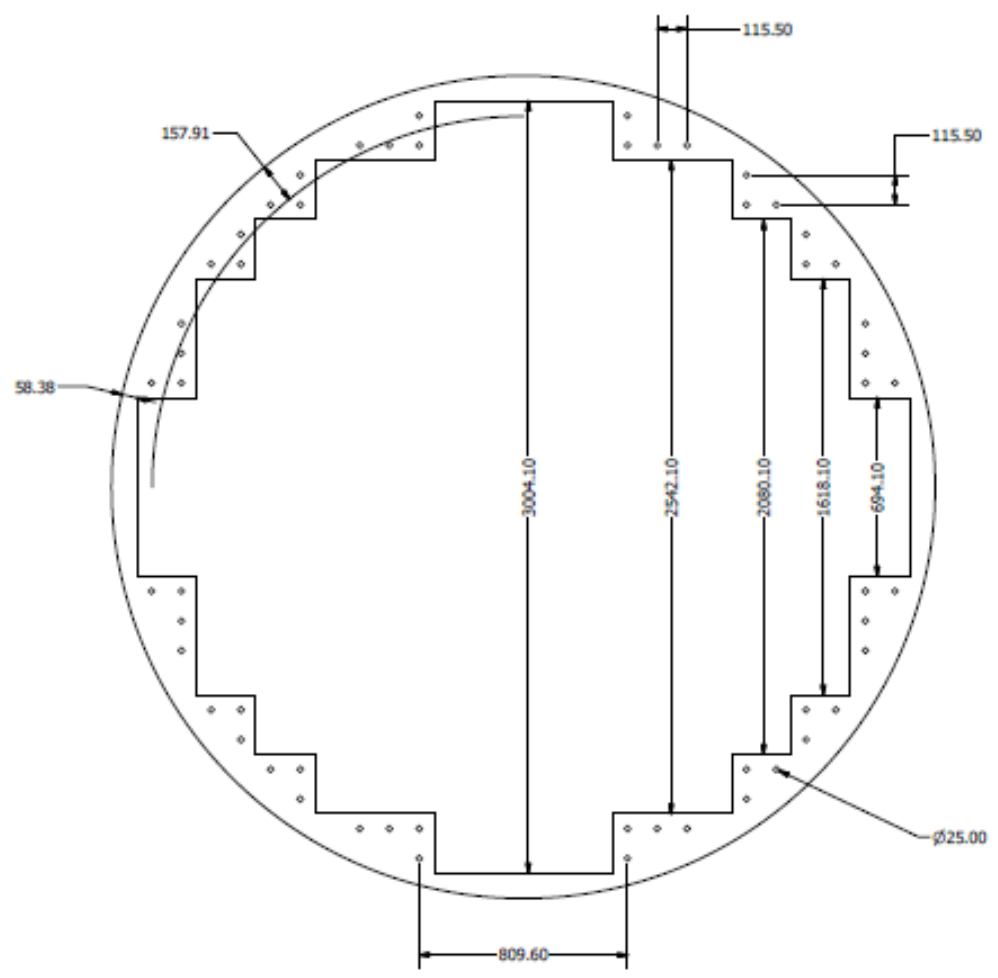

Figure 20: I2S-LWR core reflector rings.

\section{III.e.i Control Rod Drive System}

The control rod drive system is responsible for providing control rod operation and neutron management in the core. This system comprises the control rides, the control rod guide tubes, the rod control cluster assembly, the control rod drive shaft, the control rod drive mechanisms (CRDMs), the CRDM plate, and the CRDM upper plate. Each of these components is house either above or inside the core via the control rod guides in the $19 \times 19$ assemblies. 


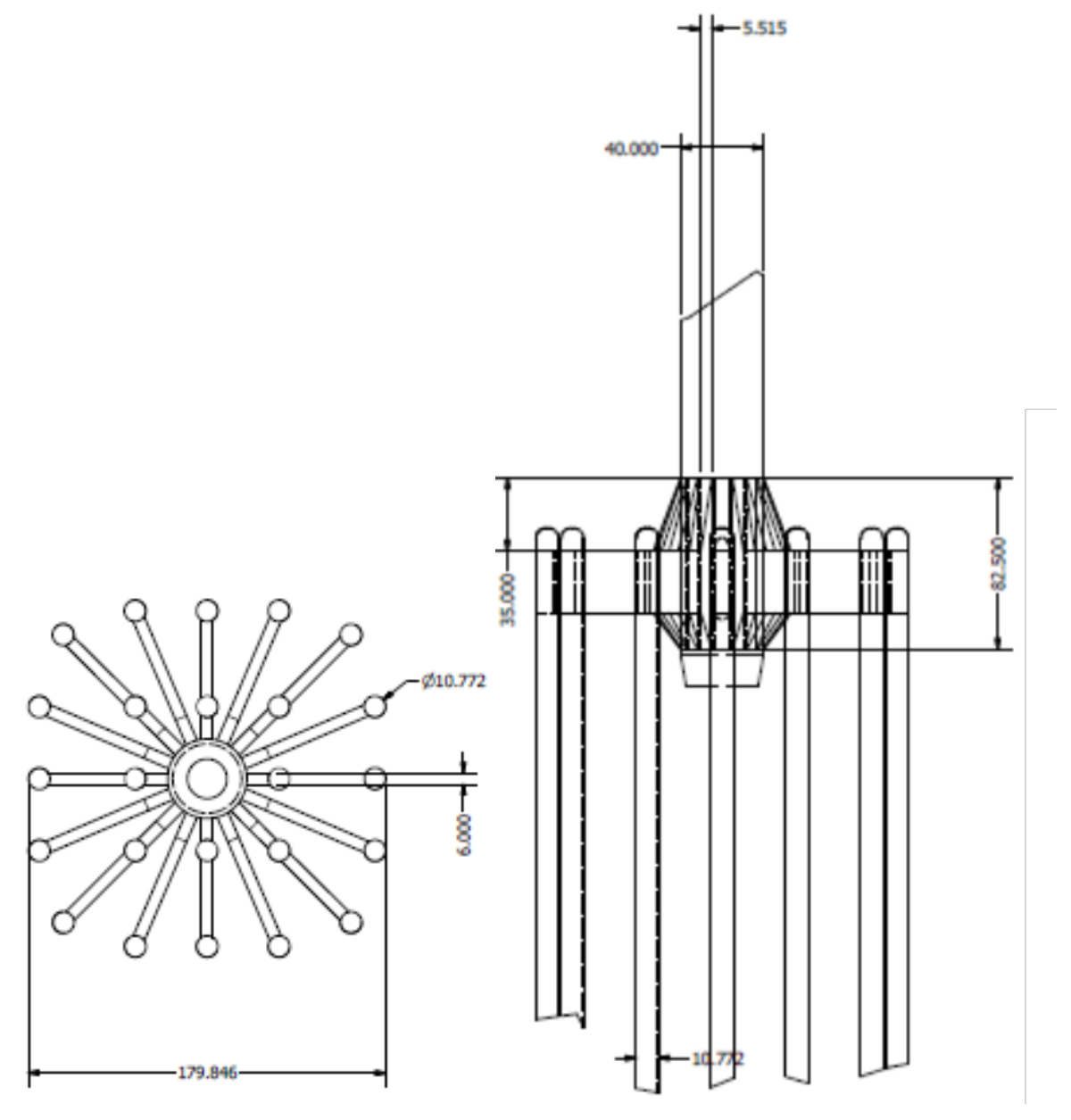

Figure 21: Illustration of the I2S-LWR (a)control rod clusters

The control rods, which are suspended and spaced via the control rod control cluster, which is illustrated in Figure 21, are inserted and withdrawn from the core as with other LWR concepts. The control rods are raised and lowered through the control rod guide tubes as the CRDM grips and maneuvers the control rod drive shaft. Unlike current concepts, however, the CRDMs are internal to the RPV, which challenges the drive mechanisms due to the harsh environment ( $2250 \mathrm{psi}$ and $600^{\circ} \mathrm{F}$ ). However, recent studies have indicated that control rod drive mechanisms can be modified to operate reliably in such an environment ${ }^{22}$.

The CRDMs, depicted in Figure 22, are supported by the CRDM plate, which also has large holes to facilitate coolant flow upward through the core barrel. The CRDM upper plate also has large holes, but inside the holes are guide pieces in order to maintain proper alignment of the control rod drive shafts as they are stepped in and out of the core. These plates are illustrated in Figure 23. 


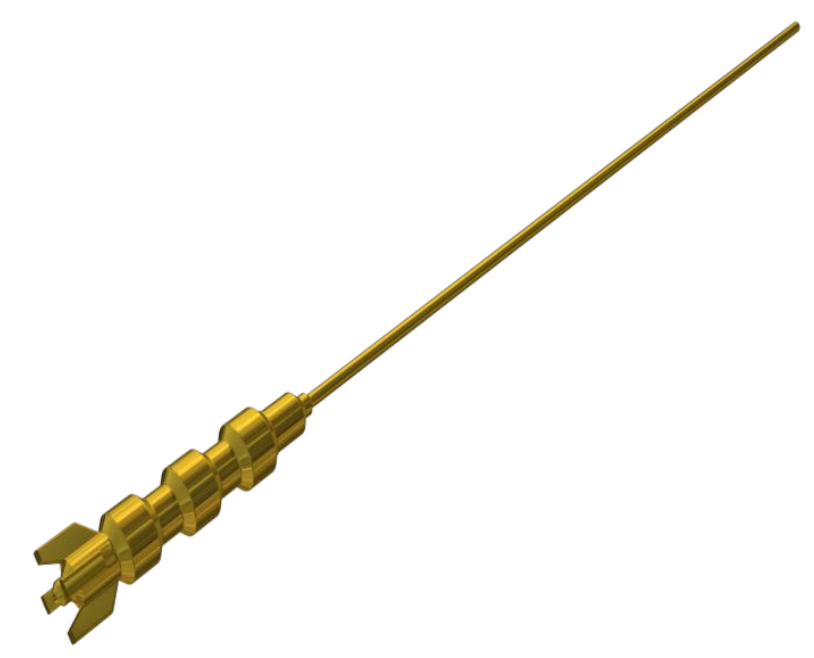

Figure 22: Control Rod Guide Mechanisms used in the I2S-LWR Concept
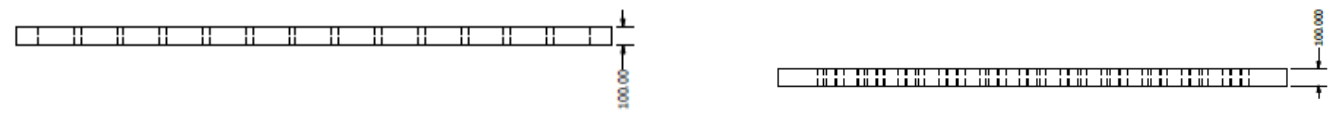

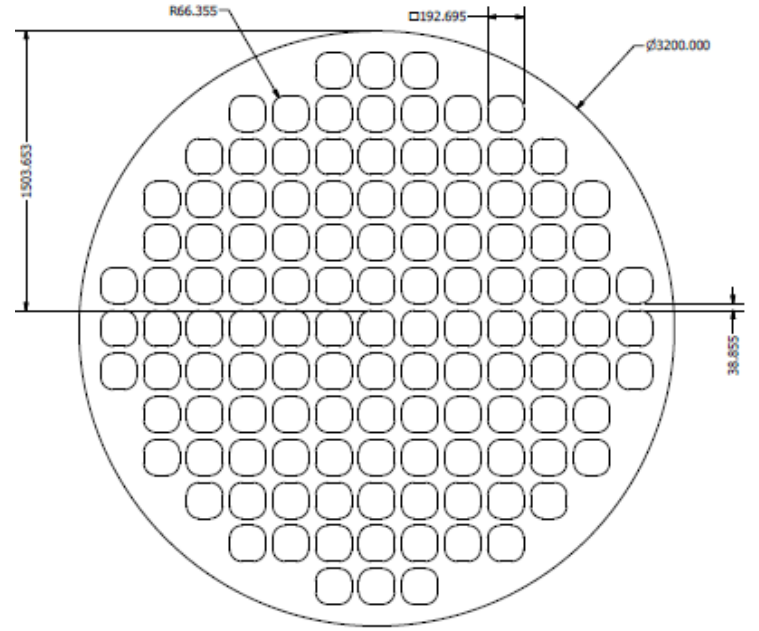

a) CRDM plate

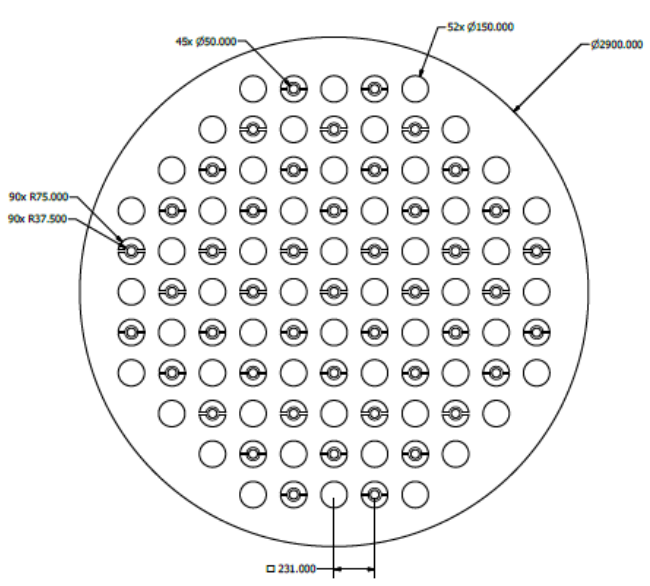

b) CRDM Upper plate

Figure 23: CRDM plates in the I'S-LWR RPV 


\section{Conclusions}

The I2S-LWR primary coolant system was design to facilitate compact systems and components while maintaining safety, economics, and ease of maintenece. The RPV is the primary component housing each of the primary systesms, and has been designed to accommodate the primary metrics indicated in section 2 of this paper. Each of the supporting systems described here has been evaluated and found acceptable in terms of both design and function for the I2S-LWR. Further design efforts will provide additional detail to the design of the primary coolant system, and will facilitate the development of a path to commercialization for the I2S-LWR.

\section{Acknowledgements}

This research was performed through the help of funding received from the DOE Office of Nuclear Energy's Nuclear Energy University Programs (NEUP). 
VI. References:

1. Kramer, D. "US Nuclear Plants Getting Fukushima-Inspired Safety Upgrades”, Physics Today, Vol. 66, Issue 1, Pg. 22-23, 2013.

2. ML113560390, "Federal Register Notice - AP1000 Design Certification Amendment, 10 CFR Part 52 (RIN 3150-Al81) NRC-2010-0131," Westinghouse Electric Company LLC., 12/22/2011.

3. C. Ye, M. G. Zheng, Y. Wang, Z. M. Qiu, "Study on the long-term passive cooling extension of AP1000 reactor", Nuclear Science and Techniques, Vol. 24, Issue 4., 2013.

4. M. C. Smith, R. F. Wright, "Westinghouse Small Modular Reactor Passive Safety System Response to Postulated Events", 12157, Proceedings of ICAPP '12, Chicago, USA, June 2013.

5. S. Boarin, M. E. Ricotti, "An Evaluation of SMR Economic Attractiveness", Science and Technology of Nuclear Installations, Vol. 2014, Article 803698, 2014.

6. Church, A. (1944). Centrifugal pumps and blowers, New York: J. Wiley.

7. Munson, B., Okiishi, T., Huebsch, W., \& Rothmayer, A. (2009). Fundamentals of fluid mechanics (7th ed.). Hoboken, NJ: J. Wiley \& Sons.

8. Reliance Motor Data Brochure, "Large AC Motors Brochure - Data Sheet", RAPS-1062, extracted July 30, 2015. www2.reliance.com/products/motors/eline/tab pages/literature tools.html.

9. Sterne, R., \& Steele, L. (1969). Steels for commercial nuclear power reactor pressure vessels. Nuclear Engineering and Design, 10(3), 259-307.

10. ASTM Data sheets, matweb.com

11. Budynas, R. G., Nisbett, J. K. (2010). Shigley's Mechanical Engineering Design, New York: McGraw-Hill.

12. Antonio C. O. Barroso, Benedito D. Baptista Fo. "Refining the design and analysis of the IRIS pressurizer", $5^{\text {th }}$ International conference on nuclear option in counties with small and medium electricity grids. Dubrovnik, Croatia, May 16-20,2004.

13. A. C. O. Barroso, B. D. Baptista Fo, I. D. Arone, L. A. Macedo, P. A. B. Sampaio, M. Moraes. IRIS pressurizer design. Proceeding of ICAPP'03, Cordoba, Spain, May 4-7,2003.

14. M. Wang, et al., "PRELIMINARY DESIGN OF THE I2S-LWR CONTAINMENT SYSTEM", submitted to Annals of Nuclear Energy, 2015.

15. M. Wang, et al., "PASSIVE DECAY HEAT REMOVAL SYSTEM DESIGN FOR THE INTEGRAL INHERENT SAFETY LIGHT WATER REACTOR (I2S-LWR)", submitted to Annals of Nuclear Energy, 2015.

16. M. J Memmott, et al., "Steam Generation system design for the Integral, Inherently safe Light Water Rector", submitted to Annals of Nuclear Energy, 2015.

17. D. Kromer, et al., "I2S-LWR Printed Channel Heat Exchanger Design and Experimental Validation", submitted to Annals of Nuclear Energy, 2015.

18. M. Wang, et al., "Passive Decay Heat Removal System Design for the Integral Inherently Safe Light Water Reactor (I2S-LWR)", submitted to Annals of Nuclear Energy, 2015.

19. P. Ferroni, et al., "Core Paper 1", submitted to Annals of Nuclear Energy, 2015.

20. P. Ferroni, et al., "Core Paper 2", submitted to Annals of Nuclear Energy, 2015.

21. M. J Memmott, A. W. Harkness, J. Van Wyk, "Westinghouse Small Modular Reactor Nuclear Steam Supply System Design", Proceedings of the International Congress on Advances in Nuclear Power Plants (ICAPP) 2012, Chicago, IL, USA, July 2012.

22. Bruce F. Allen, Gregory E. Falvo, Alexander W. Harkness, "Nuclear reactor internal hydraulic control rod drive mechanism assembly", U.S. Patent 8953732 B2, issued February 10, 2015. 